\title{
Patient portal readiness among postpartum patients in a safety net setting
}

Daryl Wieland"; Anne Gibeau'; Caitlin Dewey'; Melanie Roshto'; Hilary Frankel²

${ }^{1}$ Department of Obstetrics and Gynecology, New York City Health and Hospitals/Jacobi Medical Center, Bronx, NY, United States;

${ }^{2}$ Albert Einstein College of Medicine, Bronx, NY, United States

\section{Keywords}

Patient portal, patient engagement, prenatal care, meaningful use, electronic medical records

\section{Summary}

Background: Maternity patients interact with the healthcare system over an approximately tenmonth interval, requiring multiple visits, acquiring pregnancy-specific education, and sharing health information among providers. Many features of a web-based patient portal could help pregnant women manage their interactions with the healthcare system; however, it is unclear whether pregnant women in safety-net settings have the resources, skills or interest required for portal adoption. Objectives: In this study of postpartum patients in a safety net hospital, we aimed to: (1) determine if patients have the technical resources and skills to access a portal, (2) gain insight into their interest in health information, and (3) identify the perceived utility of portal features and potential barriers to adoption.

Methods: We developed a structured questionnaire to collect demographics from postpartum patients and measure use of technology and the internet, self-reported literacy, interest in health information, awareness of portal functions, and perceived barriers to use. The questionnaire was administered in person to women in an inpatient setting.

Results: Of the 100 participants surveyed, $95 \%$ reported routine internet use and $56 \%$ used it to search for health information. Most participants had never heard of a patient portal, yet $92 \%$ believed that the portal functions were important. The two most appealing functions were to check results and manage appointments.

Conclusions: Most participants in this study have the required resources such as a device and familiarity with the internet to access a patient portal including an interest in interacting with a healthcare institution via electronic means. Pregnancy is a critical episode of care where active engagement with the healthcare system can influence outcomes. Healthcare systems and portal developers should consider ways to tailor a portal to address the specific health needs of a maternity population including those in a safety net setting.

\section{Correspondence to:}

Daryl Wieland, MD, MS

Department of Obstetrics and Gynecology

Jacobi Medical Center

1400 Pelham Parkway S, Bldg 1, 3West, Rm 6b

Bronx, NY 10461

Phone: 17189186337

Fax: 17189186787

Email: daryl.wieland@nbhn.net
Appl Clin Inform 2017; 8: 698-709

received: December 4, 2016

accepted: April 21, 2017

published: July 5, 2017

Citation: Wieland D, Gibeau A, Dewey C, Roshto M,

Frankel H. Patient portal readiness among postpartum patients in a safety net setting. Appl Clin Inform 2017; 8: 698-709

https://doi.org/10.4338/ACI-2016-12-RA-0204 


\section{Background}

\subsection{Maternity Care}

The National Center for Healthcare Statistics (NCHS) reported almost four million births in 2015 in the United States making obstetrical care a significant reason to interact with the health system [1]. The American College of Obstetrics and Gynecology recommends women receive routine prenatal care throughout their pregnancy which includes visits every four weeks in the first seven months, then every two weeks until reaching 36 weeks and every week thereafter until delivery [2]. In addition to the need to schedule frequent appointments, an abundance of health information such as laboratory and ultrasound testing accumulates during this continuum of care. This vast amount of health data is required at the time of birth to establish treatment and delivery plans for both the mother and baby.

The mean age of a woman giving birth in the United States is 27 years old [1] and age has been reported as a strong predictor of whether adults use the internet to search for health information [3]. Using data from the National Cancer Institute's 2012 Health Information National Trends Survey (HINTS), a study of eHealth use analyzed by sociodemographic factors found that adults aged 18-34 are more than three times as likely as those 65 and older to use the internet to search for a provider or to seek answers to health questions, and females have higher eHealth utilization than males and use social networking sites at higher rates [4]. Electronically engaging maternity patients in their care offers the opportunity to enhance communication, reinforce care plans and encourage sharing of important data, functions which may be accomplished through a patient portal.

\subsection{Patient Portals}

The Meaningful Use (MU) incentive program encouraged electronic health record (EHR) adoption in the United States, providing financial incentives for healthcare organizations to implement features such as a patient portal $[5,6]$. A portal allows patient access to health data including results, visit summaries, medications, and diagnoses and an opportunity to message a provider. Despite the purported benefits of a portal, widespread adoption has not been realized and evidence on improvements in outcomes not confirmed [7-10]. Disparities in enrollment of patient portals based on race and ethnicity have been reported in primary care settings and differences in use of the portal based on age and gender have also been noted $[11,12]$. The evidence suggests that middle aged females, especially those with a chronic condition have higher rates of portal use compared to men, other age groups, and those with lower health risks [13]. In a study looking more specifically at a disadvantaged population, overall early rates of adoption were higher among patients with chronic disease who have more frequent health visits [14].

A state of the science review on patient portals and patient engagement concluded that adoption of portals is contingent upon alignment with the information needs and functional requirements of both the patients and providers [15]. Organizations, especially those in safety net settings with fewer resources, striving to meet MU objectives, struggle with how to ensure that this technology is accessible and useful to vulnerable populations [16-18]. Prior to investing significant time and resources into deploying a patient portal, healthcare institutions should seek to understand the readiness of their population to use the technology, determine if they have the necessary devices, internet access and required skills to use a portal, their level of interest in the content and what barriers could prevent them from using it. Prenatal patients represent an understudied demographic of digital natives who are familiar with technology and the internet and may be more receptive to using a portal than those populations that have been reviewed in the literature.

\section{Objectives}

The purpose of this study was to assess the stated readiness of patients in the maternity cycle to adopt a patient portal. Among postpartum patients who recently delivered a baby in an inner-city safety net setting, we aimed to: (1) determine if patients have the technical resources and skills to ac- 
cess a web-based portal, (2) gain insight into the level of interest in managing health information, and (3) identify the perceived utility of portal functions and the potential barriers to adoption.

\section{Methods}

\subsection{Setting}

This study was conducted at a single institution, New York City Health \& Hospitals/Jacobi, an innercity, publicly funded hospital in Bronx, New York. New York City Health \& Hospitals is the largest municipal hospital network in the United States. Serving a predominantly Medicaid or uninsured population, Jacobi reports approximately 25,000 outpatient obstetrical visits per year and averages 2,000 births per calendar year.

For the past 20 years, Jacobi has used an integrated electronic medical record which currently supports outpatient and inpatient clinical documentation, lab results and radiology. To meet meaningful use requirements, the institution activated a portal for patients to view inpatient admission information. The portal is not utilized in the outpatient setting, and inpatients are first introduced to the portal during admission and encouraged to sign up at that time. In the outpatient setting, to manage prenatal health information, registered prenatal patients are given a pregnancy passport, a trifold card where the provider records prenatal information, such as lab results, vital signs at each visit, and ultrasound reports.

The Albert Einstein College of Medicine Institutional Review Board approved the study and granted exempt status.

\subsection{Participants}

The participants were women 18 years of age or older currently admitted to the inpatient postpartum unit after delivery of a live infant. The hospital translator service was used to communicate with patients who did not speak English.

\subsection{Development and Administration of Questionnaire}

The interdisciplinary research team, including a clinical informatician and a member who conducted patient portal research, designed a structured questionnaire in congruence with themes identified based on review of published literature. Through a process of iterative consensus, the questionnaire was developed to measure the resources, skills, interest and perceptions, all elements that have been associated with portal adoption and patient engagement. The survey included both structured and open ended questions and took 15 minute or less to complete. After pilot testing with a small sample of patients, edits were made based on feedback. Demographic characteristics included age, parity, race/ethnicity, primary language, country of birth, education, and income.

To recruit participants, a study investigator in the postpartum unit of the hospital identified patients who had recently delivered a liveborn infant. The nature of the study was explained to the patient who verbally consented to participate or declined participation. We did not record the number of patients who declined. The hospital interpreter line was used to facilitate survey participation in the patient's preferred language for non-English speaking participants.

\subsection{Measures}

\section{Resources: Technology \& Internet Access}

To measure whether patients had the resources to use a portal, we asked what type of device (cell phone, smartphone, tablet, or computer) participants owned, the frequency and locations of internet access, and their use of an email account and social media. 


\section{Skills: Self-reported Literacy}

To assess self-reported literacy and the skill to read health information on a portal, we used a four point Likert scale to measure fluency in speaking, understanding and reading English. To measure self-reported health literacy, we used a previously validated two-question item to assess confidence in filling out medical forms and frequency of having a problem learning about medical conditions due to difficulty understanding written information [19].

\section{Attitudes: Interest in Health Information}

For interest in health information, we used a four point Likert scale to determine how often patients used the internet to find an answer about something that happened during their appointment and how often they used the internet to look up information about a health issue [20]. To understand use of the healthcare system for prenatal care and interest in personal health information, we asked about the location of prenatal care, number of different facilities used during pregnancy, whether they used the clinic's pregnancy passport to manage health information and if they read the information on the passport.

\section{Perceptions: Portal Awareness and Barriers}

To assess perceptions of a portal and its functions, we asked if patients had ever heard of a portal prior to this admission. If they answered affirmative, then they were asked what they knew about a portal and how they had used one. We explained the functions commonly offered through a patient portal and showed screenshots of a sample portal to explain the most common functions. We asked which features would be most important for them. Finally, we listed the most common potential barriers that have been previously cited in the literature and asked if those applied to them $[15,19]$. Barriers included lack of computer, lack of familiarity with internet, concerns about privacy or security, a preference for face to face communication, concerns that messages would not be answered, and not knowing the name of the provider. Patients were then invited to comment on additional perceptions with respect to portal use.

\subsection{Statistical Analysis}

We used descriptive statistics to describe the primary findings in this study. The responses to the participants' additional concerns to portal use were analyzed by two researchers for themes. The comments were coded with respect to the barriers identified in the structured portion of the survey which allowed for grouping of comments into themes which included ease of access and use of technology, a preference for face to face communication with providers, concerns for privacy and security, or the lack of barriers and advantages to a portal. All researchers then reviewed the coding to reach consensus and interpret the findings.

\section{Results}

\subsection{Participant Characteristics}

We completed questionnaires on 100 participants who were representative of the overall population of maternity patients who delivered in 2016 ( Table 1). The mean age was 28 years. The participants were ethnically and racially diverse. More than half were born outside of the United States and $30 \%$ reported a language other than English as their primary language. The hospital translator service was used to complete $16 \%$ of the surveys. The majority $(83 \%)$ had completed high school or higher education.

\subsection{Resources and Skills: Technology Use and Self-Reported Literacy}

Few subjects (1\%) reported lack of a device to access the internet and more than half (53\%) owned three devices, a smartphone, tablet and a computer. With respect to familiarity with use of the inter- 
net, $88 \%$ used a private email account and 95\% accessed the internet daily or often. Most patients (92\%) used their smartphone for internet access and either their home, library or work, and 29\% reported that the smartphone was their only source for internet access.

The majority of patients reported they had the literacy skills to read health information. Most patients (94\%) reported the ability to read English fluently or well and most (95\%) reported a high comfort level with filling out medical forms, with only $3 \%$ reporting problems understanding health information because of difficulty understanding written information ( $>$ Table 2$)$.

\subsection{Location of Healthcare \& Interest in Health Information}

Most patients (75\%) received prenatal care from the hospital's outpatient department. Forty six percent of patients used more than one facility during their prenatal episode of care illustrating the mobility of this population among healthcare providers. All the patients who had prenatal care at the facility received a pregnancy passport to manage their health information and $83 \%$ read the information on the passport. The $10 \%$ of patients who had no prenatal care or care outside the Bronx did not use a pregnancy passport to manage their health information.

With respect to use of the internet to learn about health information, $49 \%$ used the internet often or always to find out an answer to something that happened during her appointment and 56\% used the internet often or always to look up information about a health issue.

\subsection{Perceptions of Utility of Portal}

Most of the participants (76\%) had never heard of a patient portal prior to this admission. When presented with information about the portal, $92 \%$ of patients believed that the functions of the portal were important. Eighty-eight participants expressed interest in at least one of the functions that required interaction with the facility such as request an appointment $(n=63)$, message a provider $(n=61)$, review medication or request refills $(n=48)$, or correct information in the record $(n=34)$. Eighty patients selected one or more of the functions for viewing their own personal health information such as checking results $(n=68)$ or reading provider notes $(n=54)$. A majority $(61 \%)$ believed that one or more of the functions to manage their own health were important. These functions included learn about personal health issues $(n=46)$, check for preventive screening $(n=41)$, access resources for self-management $(\mathrm{n}=34)$ and download information for other providers $(\mathrm{n}=32)(\boldsymbol{\nabla}$ Figure 1).

\subsection{Perceived Barriers to Use}

Thirty-six percent of participants reported no perceived barriers to adopting a patient portal. Of those who reported barriers, $16 \%$ reported technology access barriers due to lack of a computer and $4 \%$ had no access to the internet ( $>$ Figure 2 ).

Comments related to access and usability:

"I don't have easy access to the internet."

"Is it easy to access? If not, I don't want to use it."

"Sometimes it is hard to use apps if there are too many privacy settings."

"I had a portal at another clinic. It was too much work and too hard to get into it. I forgot the username and had to do a lot to try to get it."

"They gave me a pin at the other clinic but I never got around to setting it up."

Thirty-four percent expressed concerns about privacy or security of health information on the internet. Other comments suggested less concern with health information security:

"If you can put your banking information online then why not your health?"

"Hurry up, we should have had this already. Sometimes you don't want to come [to the doctor] for just one thing...even the banks are ahead of hospitals." 
In our setting, as in many safety net settings, patients do not always have access to the same provider for each visit; however, this was not perceived as a barrier to using a portal. Only $13 \%$ believed that no one would answer their messages and only $12 \%$ stated they were not sure the name of their provider.

Thirty four percent expressed concern for the importance of the in-person relationship with a provider to explain health information.

"I don't want it to replace face to face."

"I use Google a lot to check medical information, but I would prefer to ask the doctor."

"...sometimes face to face, the doctor can explain more. On the internet if you feel confused, you stay confused, so I write it down and ask the doctor when I see them."

"I would want to have an appointment to review the test results because I am not medical and don't understand."

\section{Discussion}

Our study found that the majority of our sample of racially and ethnically diverse maternity patients attending a safety net hospital had the resources and skills necessary to adopt a patient portal. These maternity patients, many digital natives, reported a current high rate of technology use via a smartphone, tablet or computer and accessed the internet regularly through social media and to seek health information. Our findings are consistent with others who noted that many patients in a safety net setting report having the technical readiness and an interest in electronic communication with providers $[22,23]$.

Secondly, postpartum women exhibited a high level of interest in health information. Many managed their personal health information through basic means, bringing the clinic's pregnancy passport to their appointments or to other facilities. Almost half had used another facility for healthcare during their pregnancy. In other countries, such as the United Kingdom and Australia, obstetrical patients have long held a paper health record which assisted with sharing of data not only with the mother but with other providers [24, 25]. This paper health record does not exist in the United States. Paper processes are now being converted electronically which presents the opportunity for sharing information with patients and with other providers. A 2008 Canadian study found that pregnant women with internet access were more interested in accessing a web-based antenatal record when it contained personal prenatal health information than when it was for educational purposes only [26]. An Australian study specific to maternity care reviewed the use and perceptions of a portal at a large maternity hospital and found that those who used the portal viewed it favorably [27]. Both these studies occurred outside of the United States and described patients who successfully used a portal, but neither reported on those patients who did not access the portal. With ongoing challenges with interoperability and health information exchange, a portal offers the potential to allow patient in our study control of the sharing of her own health information. The utility of information sharing was not readily recognized by patients as an important function of a portal. This gap reveals an opportunity to educate patients on the use of a portal for health information exchange with other healthcare providers when indicated.

Thirdly, the majority of participants expressed interest in activities requiring interaction with the institution such as scheduling an appointment, viewing an upcoming appointment, messaging a provider, or correcting a record. Thus, the portal is not a passive internet display of electronic health data, but a forum to engage with the healthcare provider. These findings are consistent with other studies that show that more than half of patients in a safety net setting expressed interest in emailing providers and thought it would improve efficiency and avoid unnecessary visits [28]. In our study, the patients expressed confidence that messages would be answered, which suggests that policies must be implemented specific to the functions of the portal and the response by the organization, both clinically and administratively [29]. This serves as a reminder to organizations to align the utility of the portal with the expectations and needs of the user to encourage engagement and ongoing use. 
With respect to barriers to adoption, our study shows that privacy and security were not considered major barriers for portal adoption. Only a third of the patients in our study expressed concern about the security of the portal. They often access the internet through their mobile devices and in public Wi-Fi areas. This access may pose problems for the securing of information or their ability to use the portal if access is blocked in public settings, but also suggests that a portal that is compatible with a smartphone will be more useful to the users.

\subsection{Limitations}

Although our research adds useful information to the literature, we note its limitations. First, this study focused on a small, ethnically diverse population in an underserved urban setting which limits its generalizability to other populations. The urban underserved have been found to have lower rates of enrollment and usage of electronic patient portals [29]. Understanding a population with lower rates of access can provide insight into addressing the barriers to portal use and health disparities. Secondly, we created a survey for this project that has not been validated for use in any other populations or settings. We utilized convenience sampling to obtain participants on the inpatient unit, but we did not track the nonparticipants to detect differences from those who participated. While non-English speakers were included, it is possible that inadvertent selection bias resulted in more English speakers, given the need for interpreter services in a time-pressured environment. The questionnaire was conducted by interview, which meant that the answers were completed but we may have introduced social desirability bias through patients answering questions in a manner to please the interviewer. Furthermore, we did not evaluate adoption of a live portal or its actual usability. Participants reported a high level of interest in new technology but that may not translate into actual use at time of deployment.

\section{Conclusions}

Despite the limitations, this study reveals that an ethnically diverse population of maternity patients in a safety net setting are technologically prepared and eager for a patient portal tailored to their healthcare needs. Unlike previous studies on patient portal engagement, our study focused on a specific health context. Maternity care has unique characteristics that would seemingly contribute to sustained use, such as an accumulating dataset of health information which needs to be shared, specific requirements for health education to engage patients, and frequent interactions with the healthcare system to schedule appointments or message providers. Our study included patients' attitudes towards health information and perceptions of utility and barriers to portal use, thus illustrating the importance of including patient input during the development and testing of a portal so the functions align with their specific needs.

Future studies should evaluate best practices for display of prenatal content within a portal and establish links to recommended education material. Stakeholders including national provider organizations may also consider how the newborn's health information is accessed by caregivers after delivery since infancy and early childhood are yet another high-touch time when families come to the health care system at least 13 times in three years [30].

\section{Clinical Relevance Statement}

This study describes technology use and access amongst postpartum women in a safety net setting. We describe patient portal features of interest to this population that would increase adoption and utilization. These findings can be used by healthcare organizations and portal developers to tailor features and content of a portal to address the specific health needs of women in the maternity cycle of care, with the expectation of engaging more women and their families in care and improving outcomes. 


\section{Multiple Choice Questions}

Portal functions considered most important to postpartum patients in this study include:

A) Refill medication and correct information in the record

B) Review results and request an appointment

C) Download information for sharing with other providers and family

D) Check when due for a screening test and learn about personal health issues

The most commonly reported barrier to patient portal adoption was

A) Lack of a device to access the internet

B) Lack of confidence in organization to respond to messages

C) Preference for face to face communication

D) Lack of usability of a portal

\section{Answers}

Question 1: Answer b. Patients in this study reported that they wanted to review the results of their tests. The second most important function is the ability to request an appointment without having to go through the call center. The patients did not recognize the utility of using a portal to share health information which suggests a gap in knowledge that can be discussed when teaching a patient about a portal

Question 2: Answer c. About a third of patients expressed the desire to not lose the importance of face to face communication with a provider to better understand health information and not worry at home about results. Usability was not assessed in this study. Most patients had the technology resources to access a portal and expressed confidence that messages would be answered.

\section{Conflicts of Interest}

The authors report no conflicts of interests in the research.

\section{Protection of Human Subjects}

This study was approved by the Albert Einstein College of Medicine Institutional Review Board and given exempt status. 


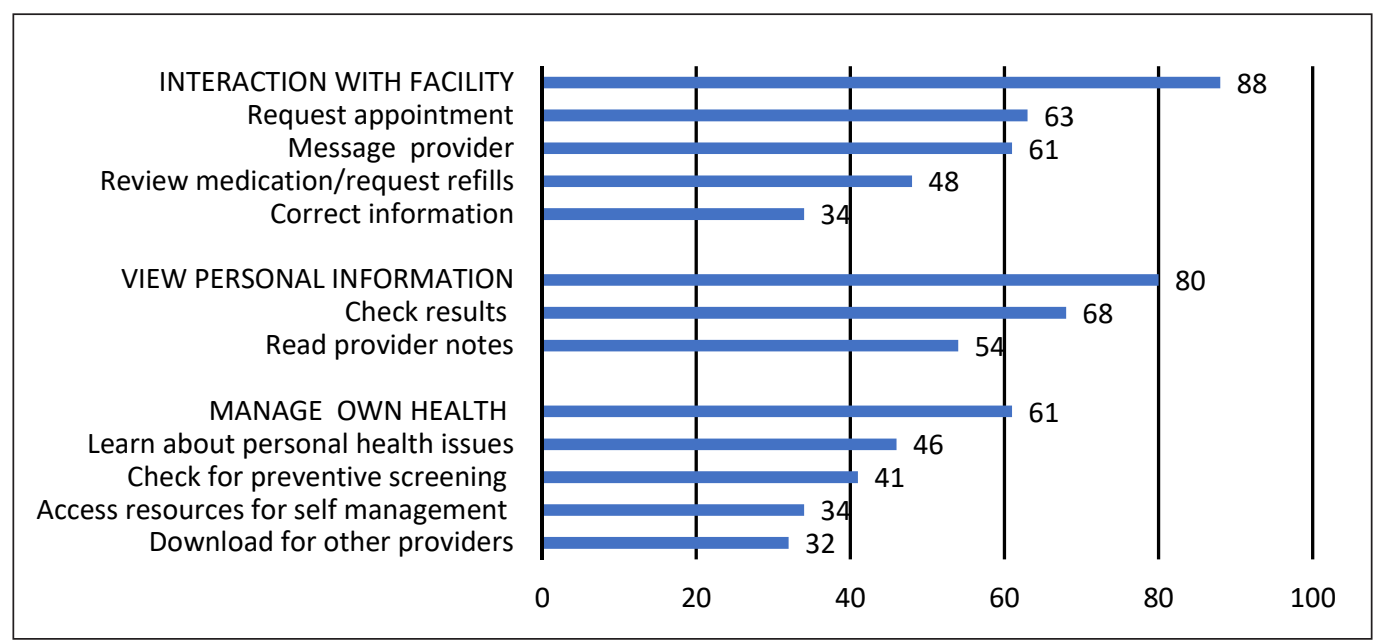

Fig. 1 Which of the functions are most appealing to you? Portal functions are grouped into three categories: functions that require interaction with the facility, functions that allow viewing of personal health information and functions that aid in the management of one's own health. Bars indicate a positive response.

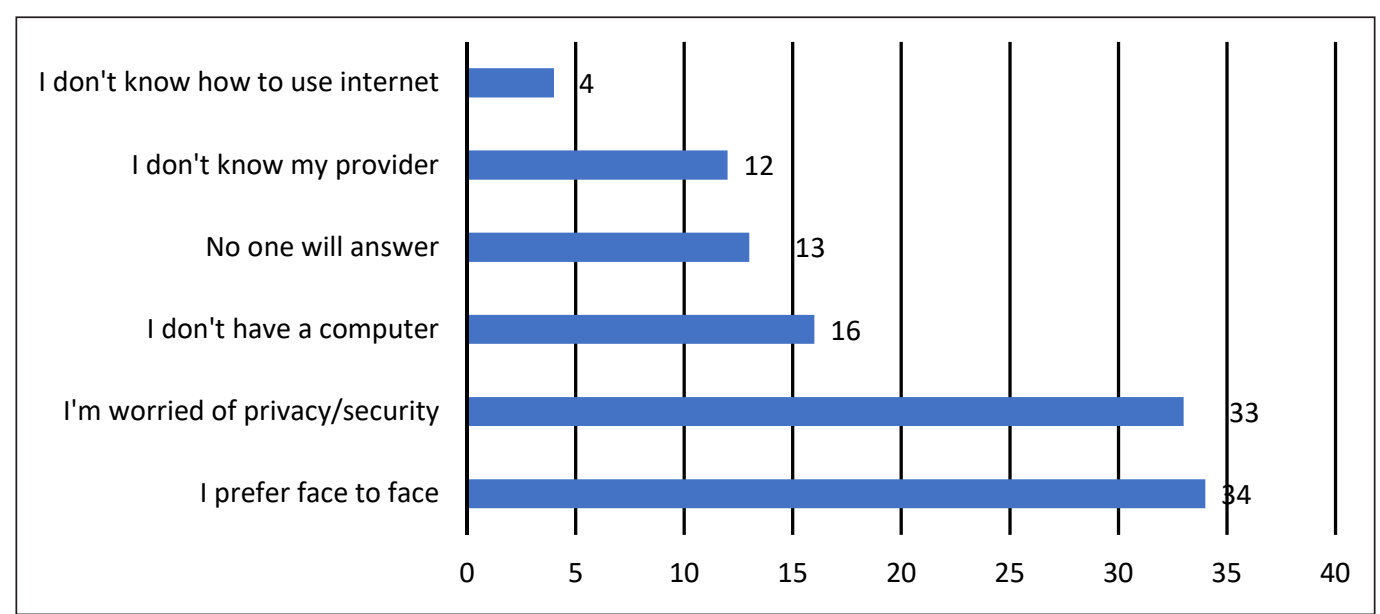

Fig. 2 Perceived barriers to portal use. 
Table 1 Patient Characteristics

\begin{tabular}{|c|c|c|}
\hline & $\begin{array}{l}\text { Study } \\
\text { Partici- } \\
\text { pants }\end{array}$ & $\begin{array}{l}\text { All De- } \\
\text { liveries } \\
2016\end{array}$ \\
\hline & $N=100$ & $N=1880$ \\
\hline $\begin{array}{l}\text { Mean age, years } \\
\text { (range) }\end{array}$ & $28(18-44)$ & $28.8(13-55)$ \\
\hline $\begin{array}{l}\text { Median parity } \\
\text { (range) }\end{array}$ & $2(1-7)$ & $1(0-10)$ \\
\hline Race & $\mathbf{n}$ & n (\%) \\
\hline $\begin{array}{l}\text { White/Caucasian/Non- } \\
\text { Hispanic }\end{array}$ & 5 & $169(9)$ \\
\hline Black/African American & 51 & $628(33.4)$ \\
\hline Asian & 10 & $204(11)$ \\
\hline Other & 2 & 699 (37) \\
\hline \multicolumn{3}{|l|}{ Ethnicity } \\
\hline Hispanic & 32 & $670(36)$ \\
\hline Non-Hispanic & 68 & $955(50.8)$ \\
\hline Not reported & 0 & $255(13.2)$ \\
\hline \multicolumn{3}{|c|}{ Birth Country / Region } \\
\hline United States & 46 & $481(25.6)$ \\
\hline $\begin{array}{l}\text { Caribbean, Central/ } \\
\text { South America }\end{array}$ & 38 & 340 (18) \\
\hline Europe & 2 & $68(4)$ \\
\hline Asia & 9 & $118(6)$ \\
\hline Africa & 5 & $127(7)$ \\
\hline No Answer & 0 & 746 (39.7) \\
\hline \multicolumn{3}{|l|}{ Primary Language } \\
\hline English & 70 & $1277(67.9)$ \\
\hline Spanish & 17 & $324(17.2)$ \\
\hline Bengali & 7 & $48(2.6)$ \\
\hline Albanian & 2 & $35(1.9)$ \\
\hline Other & 4 & $196(10.4)$ \\
\hline \multicolumn{3}{|l|}{ Education } \\
\hline Less than high school & 17 & NA \\
\hline High School grad/GED & 32 & NA \\
\hline More than high school & 51 & NA \\
\hline
\end{tabular}

Table 2 Resources: Technology access and literacy

\begin{tabular}{l|l}
\hline Technology \& Internet Access & $\%$ \\
\hline Device &
\end{tabular}

\begin{tabular}{|l|l|}
\hline Smartphone & 93 \\
\hline Tablet & 5 \\
\hline Computer or laptop & 25 \\
\hline No Internet Device & \\
\hline
\end{tabular}

\section{Frequency of internet use}

\section{Daily} 93 56

Weekly 25

Monthly/Never

Email Account

Private account

88

Shared account

3

No Email

9

Use of Social Media

Yes

81

Literacy

Read English

Fluent/Well 94

Well/Not at all

6

Confidence in filling out medical forms

Extremely

75

Quite a bit

20

Some/not at all

Difficulty in understanding written information

\begin{tabular}{|l|r|}
\hline Not at all & 67 \\
\hline Some & 29 \\
\hline Quite a bit/Extremely & 3 \\
\hline
\end{tabular}




\section{References}

1. Hamilton BE, Martin JA, Osterman MJK. Births: Preliminary Data for 2015. National Vital Statistics Reports 2016;65(3). Hyattsville, MD: National Center for Healthcare Statistics. 2015. Available from: http://www.cdc.gov/nchs/data/nvsr/nvsr65/nvsr65_03.pdf

2. American Academy of Pediatrics and American College of Obstetricians and Gynecologists. 2012. Guidelines for perinatal care. Elk Grove Village, IL: American Academy of Pediatrics.

3. Fox S, Duggan M. Pew Research Center Health Online 2013. Available at http://www.pewinternet.org/ files/old-media//Files/Reports/PIP_HealthOnline.pdf.

4. Kontos E, Blake KD, Chou WYS, Prestin A. Predictors of eHealth Usage: Insights on the Digital Divide from the Health Information Trends Survey 2012. J Med Internet Res 2014; 16(7): e172.

5. "HITECH Ace Enforcement Interim Final Rule." https://www.hhs.gov/hipaa/for-professionals/specialtopics/HITECH-act-enforcement-interim-final-rule/. Accessed Dec 1, 2016.

6. Office of the National Coordinator. What is a patient portal? URL: http://www.healthit.gov/providers-pro fessionals/faqs/what-patient-portal [accessed November 2016] [WebCite Cache].

7. Carrell D, Ralston JD. Variation in Adoption Rates of a Patient Web Portal with a Shared Medical Record by Age, Gender, and Morbidity Level. AMIA Annual Symposium Proceedings 2006; 2006: 871.

8. Zhou YY, Garrido T, Chin HL, Wiesenthal AM, Liang LL. Patient access to an electronic health record with secure messaging: impact on primary care utilization. Am J Manag Care 2007; 13(7): 418-424.

9. Kruse CS, Bolton K, Freriks G. The effect of patient portals on quality outcomes and its implications to meaningful use: A systematic review. J Med Internet Res 2015; 17(2): e44.

10. Griffin A, Skinner A, Thornhill J, Weinberger M. Patient portals: Who uses them? What features do they use? And do they reduce readmissions? Appl Clin Inform 2016; 7:489-501.

11. Sarkar U, Karter AJ, Liu JY, Adler NE, Nguyen R, Lopez A, Schillinger D. The Literacy Divide: Health Literacy and the Use of an Internet-Based Patient Portal in an Integrated Health System-Results from the Diabetes Study of Northern California (DISTANCE). J Health Comm 2010; 15(2): 183-196.

12. Goel MS, Brown TL, Williams A, Hasnain-Wynia R, Thompson J, Baker DW. Disparities in Enrollment and Use of an Electronic Patient Portal. J Gen Intern Med 2011; 26(10): 1112-1116.

13. Goldzweig CL, Orshansky G, Paige NM, . Electronic patient portals: evidence on health outcomes, satisfaction, efficiency, and attitudes: a systematic review. Ann Intern Med 2013;159(10): 677-687.

14. Ancker JS, Barrón Y, Rockoff ML, Hauser D, Pichardo M, Szerencsy A, Calman N. Use of an Electronic Patient Portal Among Disadvantaged Populations. J Gen Intern Med 2011; 26(10): 1117-1123.

15. Irizarry T, Dabbs AD, Curran CR. Patient Portals and Patient Engagement: A State of the Science Review. J Med Internet Res 2015; 17(6): e148.

16. Hilton JF, Barkoff L, Chang O, Halperin L, Ratanawongsa N, Sarkar U, Leykin Y, Munoz RF, Thorn DH, Kahn JS. A Cross-Sectional Study of Barriers to Personal Health Record Use among Patients Attending a Safety-Net Clinic. PLoS ONE 2012; 7(2): e31888.

17. Goel MS, Brown TL, Williams A, Cooper AJ, Hasnain-Wynia R, Baker DW. Patient reported barriers to enrolling in a patient portal. J Am Med Inform Assoc 2011; 18: i8-i12.

18. Dhanireddy S, Walker J, Reisch L, Oster N, Delbanco T, Elmore JG. The urban underserved: attitudes toward gaining full access to electronic medical records. Health Expect 2014; 17(5): 725-732.

19. Chew LD, Bradley KA, Boyko EJ. Brief questions to identify patients with inadequate health literacy. Fam Med 2004; 36(8): 588-594.

20. Nelson DE, Kreps GL, Hesse BW, Croyle RT, Willis G, Arora NK, Rimer B, Viswanath KV, Weinstein N, Alden S. The Health Information National Trends Survey (HINTS): development, design, and dissemination. J Health Commun 2004; 9 (5): 443-460.

21.Lyles C, Allen JY, Poole D, Tieu L, Kanter MH, Garrido T. I want to keep the personal relationship with my doctor: Understanding barriers to portal use among African Americans and Latinos. J Med Internet Res 2016; 18(10)e263.

22.Schickedanz A, Huang D, Lopez A, Cheung E, Lyles CR, Bodenheimer T, Sarkar U. Access, interest, and attitudes toward electronic communication for health care among patients in the medical safety net. J Gen Intern Med 2013; 28(7): 914-920.

23. Saunders MR, Winters P, Fortuna RJ, Mendoza M, Berliant M, Clark L, Fiscella K. Internet access and patient portal readiness among patients in a group of inner-city safety-net practices. J Ambulatory Care Manage 2013; 36(3): 251-259.

24. Hawley G, Janamian T, Jackson C, Wilkinson SA. In a maternity shared-care environment, what do we know about the paper hand-held and electronic health record: a systematic literature review. BMC Pregnancy and Childbirth 2014; 14: 52. 
25. Hawley G, Jackson C, Hepworth J, Wilkinson SA. Sharing of clinical data in a maternity setting: How do paper hand-held records and electronic health records compare for completeness? BMC Health Services Research 2014; 14: 650.

26. Shaw E, Howard M, Chan D, Waters H, Kaczorowski J, Price D, Zazulak J. Access to Web-Based Personalized Antenatal Health Records for Pregnant Women: A Randomized Controlled Trial. J Obstet Gynaecol Can 2008; 30(1): 38-43.

27. Forster M, Dennison K, Callen J, Georgiou A, Westbrook J. I. Maternity patients' access to their electronic medical records: use and perspectives of a patient portal. Health Information Management Journal 2015; 44(1): 4-11.

28. Wallace LS, Angier H, Huguet N, Gaudino JA, Krist A, Dearing M, Killerby M, Marino M, DeVoe JE. Patterns of Electronic Portal Use among Vulnerable Patients in a Nationwide Practice-based Research Network: From the OCHIN Practice-based Research Network (PBRN). Journal of the American Board of Family Medicine: JABFM 2016; 29(5): 592-603.

29. Osborn CY, Rosenbloom ST, Stenner SP, Anders S, Muse S, Johnson KB, Jirjis J, Jackson GP. MyHealthAtVanderbilt: policies and procedures governing patient portal functionality. J Am Med Inform Assoc 2011; 18: i18-i23.

30. American Academy of Pediatrics. Recommendations for preventive pediatric healthcare. Available from https://www.aap.org/en-us/documents/periodicity_schedule.pdf. Accessed March 19, 2017. 